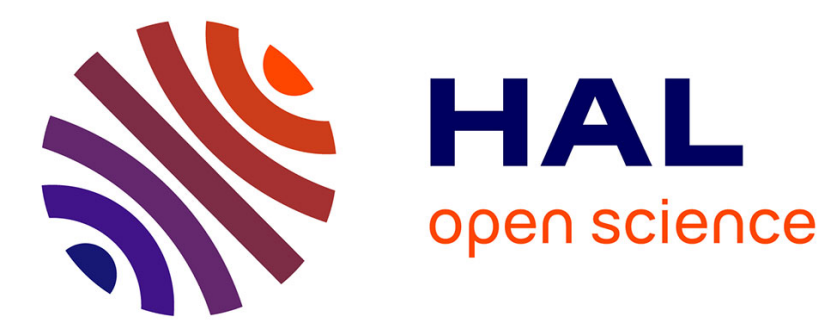

\title{
Van der Waals interactions in the lattice of metallic chains
}

\author{
S. Barišić, A. Bjeliš
}

\section{To cite this version:}

S. Barišić, A. Bjeliš. Van der Waals interactions in the lattice of metallic chains. Journal de Physique Lettres, 1983, 44 (9), pp.327-332. 10.1051/jphyslet:01983004409032700 . jpa-00232199

\section{HAL Id: jpa-00232199 https://hal.science/jpa-00232199}

Submitted on 1 Jan 1983

HAL is a multi-disciplinary open access archive for the deposit and dissemination of scientific research documents, whether they are published or not. The documents may come from teaching and research institutions in France or abroad, or from public or private research centers.
L'archive ouverte pluridisciplinaire HAL, est destinée au dépôt et à la diffusion de documents scientifiques de niveau recherche, publiés ou non, émanant des établissements d'enseignement et de recherche français ou étrangers, des laboratoires publics ou privés. 
Classification

Physics Abstracts

$71.45 \mathrm{~N}-61.50 \mathrm{~L}$

\title{
Van der Waals interactions in the lattice of metallic chains
}

\author{
S. Barišić \\ Department of Physics, Faculty of Science, P.O.B. 162, Zagreb, Croatia, Yugoslavia
}

and A. Bjeliš

Institute of Physics of the University, P.O.B. 304, Zagreb, Croatia, Yugoslavia

(Reçu le 16 novembre 1982, accepté le 11 mars 1983)

\begin{abstract}
Résumé. - Nous calculons l'énergie de cohésion d'un réseau de chaînes métalliques en la développant par rapport à l'interaction coulombienne, supposée petite par rapport aux intégrales de transfert intrachaînes. Ce développement contient les interactions de Van der Waals, associées aux polarisabilités métalliques des chaînes. Pour un couplage interchaîne suffisamment fort (mais en supposant les termes de diffusion vers l'arrière négligeables par rapport à ceux de diffusion avant), l'énergie de Van der Waals, gagnée en passant du réseau HMTTF-TCNQ au réseau TTF-TCNQ, est comparable à la perte correspondante de l'énergie de Madelung associée à la distribution homogène des charges le long des chaînes.
\end{abstract}

\begin{abstract}
The cohesive energy of a lattice of metallic chains is calculated as a perturbation expansion of the Coulomb interaction, which is assumed small relative to the intrachain overlap integrals. The expansion contains the Van der Waals like interactions associated with the metallic polarizabilities of the chains. For sufficiently large forward interchain coupling (backward contribution neglected), the Van der Waals energy gained on going from the HMTTF-TCNQ to TTF-TCNQ lattice competes with the corresponding loss of the Madelung energy of the charges homogeneously distributed along the chains.
\end{abstract}

Organic metals belonging to the TTF-TCNQ family which have been extensively studied in recent years crystallize in a segregated stack structure [1]. Like molecules TTF or HMTTF (F) and TCNQ $(Q)$ form conducting chains along the $b$-axis. The organization of the chains in the $a-c$ plane differs however according to the material. E.g. figure $1 a$ shows schematically the $a-c$ plane in TTF-TCNQ and figure $1 b$ the same plane in HMTTF-TCNQ. Due to the transfer $[2,3]$ of a fractional number $\rho$ of electrons per donor or acceptor molecule $(\rho \approx 0.6-0.7)$ the chains are charged. This charge is evenly distributed along the chain in the metallic phase, but at low enough temperatures $\left(10-10^{2} \mathrm{~K}\right)$ the $2 k_{\mathrm{F}}, 4 k_{\mathrm{F}}$ modulations (CDWs) may appear $[2,3]\left(k_{\mathrm{F}}=\frac{\pi}{b} \rho\right)$. The condensation of these superstructures has little or no effect on the charge transfer (i.e. on $k_{\mathrm{F}}$ ) itself $[2,3]$ and on the lattice parameters [4].

In most previous attempts to understand the occurrence of the segregated stack structures attention was focused on the balance between the ionization - affinity energy $E_{\mathrm{IA}}$ and the Madelung energy $E_{\mathrm{M}}[5,6]$. It turned out $[5,6]$ that with an homogeneous charge distribution 


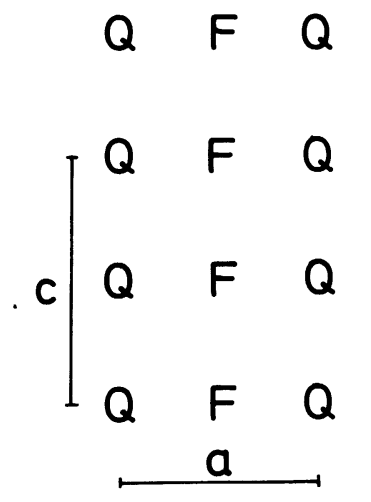

(a)

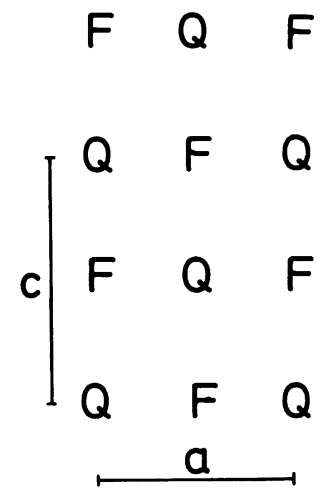

(b)

Fig. 1. - Schematic packing of chains in the TTF-TCNQ (a) and HMTTF-TCNQ (b) structure.

along the chain the energy gain $E_{\mathrm{M}}$ was insufficient to offset the loss $E_{\mathrm{IA}}$. This energy imbalance may be improved by assuming $[7,8]$ that the transfered charges are not uniformly distributed, but form Wigner lattices along the chains. However, it should be realized that these lattices represent extreme cases of the $2 k_{\mathrm{F}}, 4 k_{\mathrm{F}}$ CDWs. If their formation were responsible for the stability of the charge transfer, their disappearance at low temperatures should be accompanied by significant variations of the charge transfer (i.e. of $2 k_{\mathrm{F}}$ ) and of the lattice parameters. This is at variance with the experimental results mentioned above. Besides, arguments based solely on the Madelung and the ionization energies should lead to full charge alternation in all directions (i.e. to the Q-F alternation). A different mechanism is therefore required to understand the stability of the segregated stack structures.

In this respect Friedel has pointed out [9] that the dispersive forces (Van der Waals interactions, interactions of permanent dipoles etc.) favour the close packing of like molecules. His argument can be reproduced in a few words : consider [10] the two molecular distributions of figures $1 a, b$. Going from figure $1 b$ to figure $1 a$ involves replacing the two Q-F bonds by a Q-Q and an F-F bond. Assume for example that those are the Van der Waals (VdW) bonds. Keeping all other parameters constant and using the London approximation for the Van der Waals energy, the corresponding energy change is

$$
\Delta E_{\mathrm{VdW}} \sim 2 \alpha_{\mathrm{Q}} \alpha_{\mathrm{F}}-\left(\alpha_{\mathrm{Q}}^{2}+\alpha_{\mathrm{F}}^{2}\right)=-\left(\alpha_{\mathrm{Q}}-\alpha_{\mathrm{F}}\right)^{2} \lesssim 0,
$$

as required. Here $\alpha$ 's denote the polarizabilities of the $Q, F$ molecules.

The argument (1), if carried to its extreme, leads to the full Q-F segregation. As the opposite extreme (full Q-F alternation) follows from the Madelung argument, it is tempting to attribute the observed lattices to the fine balance between the dispersive and the Madelung forces : in contrast to the Madelung forces the VdW forces prefer the TTF-TCNQ lattice of figure $1 a$ to the HMTTF-TCNQ lattice of figure $1 b$. Unfortunately, the homogeneous charge distribution along the chain is not a natural consequence of the model based exclusively on these two types of forces. First, the molecular polarizabilities seem to be too small to make the cohesive energy of this structure positive [7]. Second, the model tends to prefer the formation of the strong Wigner lattices along the chain to the homogeneous charge distribution.

On the other hand, the homogeneous charge distribution is a natural ingredient of the model of metallic chains. Although widely accepted in the description of the low temperature properties [11-13], this model was not previously considered successful [5, 6] in the evaluations of the 
cohesive energy. The reason is that the band energy $E_{\mathrm{B}}$ tends to be small with respect to $E_{\mathrm{IA}}$. However, we wish to point out here that there are other energy contributions arising from the metallic chains, which favour the observed lattices. In particular, we will show that the metallic chains act as polarizable units and the notion of $\mathrm{VdW}$ interactions between chains can be retained. The organization of the $a-c$ plane in thus still covered by Friedel's equation (1), upon identifying the $\alpha$ 's with the polarizabilities of the metallic chains.

The condition for the validity of equation (1) in such an interpretation is that the interchain interactions are weak. Unfortunately, this requirement is not very well fulfilled in the actual materials. The Coulomb forces are long ranged, i.e. of the same order of magnitude within and between the chains. Their order of magnitude can be estimated [13] from the intra-band plasmon frequency $\omega_{0}$. As the latter turns out to be of the same order of magnitude as the band-width [14], we are encountering the intermediate coupling regime. Nevertheless, in order to conserve explicitly the attractive features associated with equation (1) we shall scale down all the interactions, rather than assume artificially that only the interchain couplings are weak. Solving the weak coupling problem we shall see that it is physically reasonable to extrapolate the results to moderate couplings.

The method which bridges best between weak and strong coupling limit is the tight-binding (TB) method [15, 16]. From the point of view of Coulomb forces this method is related [16] to the Wigner-Seitz (W-S) approach [17]. The TB wave-functions satisfy the W-S boundary conditions and are calculated on each site with the appropriate ionic potential. Thereby they tend to anticipate the results of the RPA calculation [17].

In the TB/W-S scheme the cohesive energy is decomposed as

$$
E_{\mathrm{C}}=E_{\mathrm{IA}}+E_{\mathrm{EC}}+E_{\mathrm{B}}+E_{\mathrm{SFE}}+E_{\mathrm{HF}}+E_{\mathrm{COR}}
$$

$E_{\mathrm{IA}}$ and $E_{\mathrm{B}}$ have been already defined. $E_{\mathrm{EC}}$ involves all the energies not related to the band formation and the charge transfer (includes e.g. "steric " effects). $E_{\mathrm{SFE}}$ is the self-energy of the valence charge on the site. It brings us back from the W-S to the Hartree scheme, which is the usual starting point for the calculation of the Hartree-Fock energy $E_{\mathrm{HF}}$ and the correlation energy $E_{\mathrm{COR}}$. In ordinary metals the two last terms in equation (2) roughly cancel out $E_{\mathrm{SFE}}$, justifying the use of the " single electron " W-S term $E_{\mathrm{B}}$ [17]. The situation is somewhat different here. $E_{\mathrm{SFE}}$ is the local (on-site, on-molecule) term, whereas $E_{\mathrm{HF}}$ and $E_{\mathrm{COR}}$ contain the interchain terms, which we believe to be responsible for the choice between the two lattices in figure 1 .

A few low-order diagrams of the weak-coupling theory for $E_{\mathrm{HF}}+E_{\mathrm{COR}}$ are shown in figure 2. The term $E_{\mathrm{H}}$ in figure $2 a$ is the Hartree term, which is identical to the Madelung energy $E_{\mathrm{M}}$. Indeed, the loops in figure $2 a$ denote the homogeneous charge distributions on the chains $n$ and $m$ which interact through the Coulomb interaction $V_{n m}(q=0) . E_{\mathrm{F}}$ of figure $2 b$ is the Fock term. This is an on-chain term, which cancels for the energy difference of the two lattices shown in figures $1 a, b$. The term which is of particular interest here is shown in figure $2 c$. For chains at distances $R_{n m}$ we get from this figure

$$
\begin{aligned}
E_{\mathrm{VdW}} & =\frac{1}{2} \sum_{n m} E_{\mathrm{VdW}}(n, m), \quad R_{n m} \lesssim k_{\mathrm{TF}}^{-1}, \\
E_{\mathrm{VdW}}(n, m) & =-\frac{1}{4 \pi} \sum_{|q|>k_{\mathrm{TF}}} V_{n m}^{2}(q) \int_{0}^{\infty} \operatorname{Im}\left[\alpha_{n}(q, \omega) \alpha_{m}(q, \omega)\right] \mathrm{d} \omega .
\end{aligned}
$$

We note immediately that the VdW energy between two chains [18] $E_{\mathrm{Vdw}}(n, m)$ has the same general structure as the terms in equation (1). The diagram $2 c$ (i.e. Eq. (3)), which is usually interpreted as describing the short range $\left(R \lesssim k_{\mathrm{TF}}^{-1}\right)$ forces between electrons of the jellium [19] charac- 


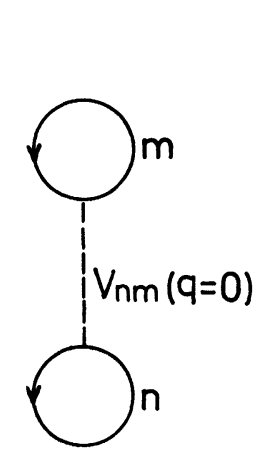

(a)

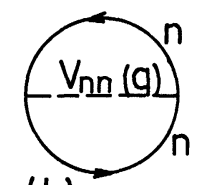

(b)

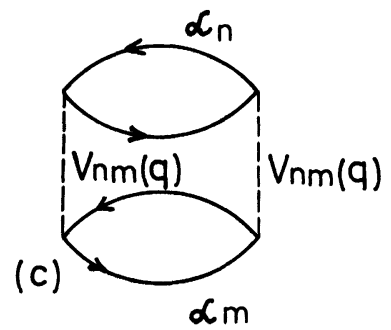

Fig. 2. - Diagrams representing respectively Hartree (i.e. Madelung) (a) Fock (b) and Van der Waals (c) terms in the cohesive energy.

terized by the Thomas-Fermi wave-vector $k_{\mathrm{TF}}$, has in our TB limit the meaning of the VdW energy of a pair of metallic chains.

The cut-off $k_{\mathrm{TF}}$ describes the screening of the Coulomb interactions by the lattice of metallic chains [20]. It is given by [13]

$$
k_{\mathrm{TF}}^{2} a c=16 \pi n_{\mathrm{F}} e^{2} / b=4 \pi^{2} n_{\mathrm{F}}^{2} \omega_{0}^{2} a c / b^{2}<1,
$$

where $n_{\mathrm{F}}$ is the density of electron states at the Fermi level. For wave-vectors smaller than $k_{\mathrm{TF}}$ the perturbative diagram of figure $2 c$ is not sufficient, and even in the weak-coupling limit the bare interactions must be replaced at least by their RPA screened values. This range contributes to the cohesive energy through the zero point motion of the plasmons, strongly renormalized by the long-range Coulomb forces. The maximum frequency of those plasmons is $\omega_{0}$ of equation (4). However, according to this equation, the phase space in which the Coulomb screening is essential, is small in the weak coupling limit $n_{\mathrm{F}} \omega_{0}<1$. Its contribution can thus be left out with respect to that of equation (3). This is analogous to the $r_{\mathrm{s}}$ expansion [19] of the $3 \mathrm{~d}$ jellium model.

Let us turn now to the integration over $q, \omega$ in equation (3). In the interchain terms $n \neq m$, which are of primary interest here, the Coulomb coupling $V_{n m}(q)$ is appreciable for $q<a^{-1}$, $c^{-1} \lesssim k_{\mathrm{F}}$. In other words the interchain backward scattering is weak, and we shall neglect it here. This cuts-out the logarithmic singularities of the bubbles in our low-order diagram $2 c$. For essentially the same reason we are omitting altogether the many-body corrections, related to the backward scattering and discussed elsewhere in the same physical model [13]. All this is consistent with our general idea that the condensation of CDW superstructures (Wigner lattices) plays a minor role in the cohesion of the main lattice. With all this in mind we get from equation (3)

$$
E_{\mathrm{VdW}}(n, m) \approx-\frac{e^{4}}{\pi^{2} R_{n m}^{2}} \cdot \frac{n_{\mathrm{F}}^{(m)} n_{\mathrm{F}}^{(m)}}{n_{\mathrm{F}}^{(n)}+n_{\mathrm{F}}^{(m)}}, \quad 0<R_{n m}<k_{\mathrm{TF}}^{-1} .
$$

Here $n_{\mathrm{F}}^{(i)}$ denote the densities of states, determined in the usual way by the hopping integrals and the band filling $\rho$. Equation (5) shows that in contrast to the usual VdW energy $E_{\mathrm{Vdw}}(n, m)$ is not a rapidly decreasing function of $R_{n m}$. The reason is that the dipoles along the chains important in equation (5) are of lengths $q^{-1}$ which are larger than the interchain distance $R_{n m}$. Thus instead of going straightforwardly to the first-neighbour argument of equation (1), we prefer to perform the lattice sums first. 
In order to bring this problem to qualitatively the same level of approximation as that used until now, we shall replace the actual lattice by its orthorhombic counterpart with interchain distances equal to $a / 2, c / 2$. The general form of the lattice sum(3) for both lattices of figures $1 a, b$ is

$$
E_{\mathrm{VdW}} \approx-\frac{n_{\mathrm{F}} e^{4}}{a c} C_{1} \log \left[1 / C_{2} k_{\mathrm{TF}} \sqrt{a c}\right]
$$

where $n_{\mathrm{F}} \simeq \max \left(n_{\mathrm{F}}^{\mathrm{Q}}, n_{\mathrm{F}}^{\mathrm{F}}\right)$, while the constants $C_{1}$ and $C_{2}$ depend weakly on the ratio $a / c$, and are of the order unity. Equation (6) shows that the long-range interactions dominate $E_{\mathrm{Vdw}}$ in the weak-coupling limit. This justifies the use of the point charge approximation [21], which is implicit in all our calculations.

The long-range features disappear however from the difference of the $\mathrm{VdW}$ energies for the two lattices of figures $1 a, b$. The corresponding lattice sum can be evaluated exactly to give

$$
\Delta E_{\mathrm{VdW}}=\frac{e^{4}}{2 \pi a c} \cdot \frac{\left(n_{\mathrm{F}}^{\mathrm{Q}}-n_{\mathrm{F}}^{\mathrm{F}}\right)^{2}}{n_{\mathrm{F}}^{\mathrm{Q}}+n_{\mathrm{F}}^{\mathrm{F}}} \log \left(1-k^{2}\right),
$$

where $k$ is related to $c / a$ through the elliptic [22] integral $K$

$$
\frac{c}{a}=\frac{K\left(\sqrt{1-k^{2}}\right)}{K(k)} .
$$

For $\pi a / 2 c \gtrsim 1$, satisfied in TTF-TCNQ, $\log \left(1-k^{2}\right) \approx-\pi a / c$.

Equation (7) agrees qualitatively with equation (1), replacing $\alpha_{F}$ and $\alpha_{Q}$ by the metallic polarizabilities proportional to $n_{\mathrm{F}}^{\mathrm{F}}$ and $n_{\mathrm{F}}^{\mathrm{Q}}$ respectively. It also supplies the missing prefactor, which is not very accurate however, because the point-charge approximation is not accurate at short distances.

Nevertheless it is interesting to compare $\Delta E_{\mathrm{Vdw}}$ to the corresponding Madelung (Hartree) energy evaluated within this same point-charge approximation. The latter can be found from the closed expressions of reference [21]. The best convergence for $E_{M}$ of a given lattice is obtained by performing first the summation along the $a$-direction, where the charge alternation occurs in both lattices of figures $1 a, b$. The next step is then devoted to the summation of the interactions between the fictitious chains in the $a$-direction. In this way we get

$$
\begin{aligned}
E_{\mathbf{M}}^{\mathrm{T}, \mathrm{H}}=\frac{4 N \rho^{2} e^{2}}{a}\left\{-\log 2+\sum_{n^{2}{ }_{2}+n^{2}{ }_{3} \neq 0} \mathrm{e}^{i v \pi} \sum_{\mathcal{N}=0, \pm 1, \ldots} K_{0}\left\lceil\frac{2 \pi}{a}(1+2 \mathcal{N}) \times\right.\right. & \left.\left.\times\left(b^{2} n_{2}^{2}+(c / 2)^{2} n_{3}^{2}\right)^{1 / 2}\right]\right\},
\end{aligned}
$$

omitting the on-site term, but retaining for convenience the other intrachain terms. Here $K_{0}$ denotes the Bessel function [22], while $v=0$ for TTF-TCNQ and $v=n_{3}$ for HMTTF-TCNQ. $E_{\mathrm{M}}$ is dominated by the long-range forces (i.e. point-charge approximation is appropriate) for $b \ll a / 2 \pi, c / 2$ when $K_{0}$ 's involving $b$ are becoming logarithmically singular. However in TTFTCNQ the anisotropy comes from the TB overlap integrals rather than from the lattice anisotropy and this condition is not satisfied. In fact for the actual values of lattice parameters all $K_{0}$ functions in equation (9) are better approximated by the asymptotic expansion $K_{0}(x) \sim$ $\mathrm{e}^{-x} / \sqrt{x}$ than by the logarithmically singular behaviour of the opposite small $b$ limit. We are thus evaluating $E_{\mathrm{M}}$ with roughly the same degree of accuracy as $\Delta E_{\mathrm{Vdw}}$. From this point of view there is no essential difference between $E_{\mathrm{M}}$ and $\Delta E_{\mathrm{M}}$, which is given by

$$
\Delta E_{\mathrm{M}} \approx+\frac{16 e^{2} \rho^{2}}{b} \exp \left(-\frac{\pi c}{a}\right) \text {. }
$$


It is somewhat simpler to discuss the relative stability of the two lattices of figures $1 a, b$ rather than the stability of each lattice separately. The reason is that the discussion of the relative stability does not involve the intrachain terms and that it deals with the qualitative effect. Let us thus consider the ratio of $\left|\Delta E_{\mathrm{VdW}}\right|$ to $\left|\Delta E_{\mathrm{M}}\right|$. Not surprinsingly for the weak-coupling limit this ratio is of the order of $n_{\mathrm{F}}^{2} \omega_{0}^{2}$, i.e. smaller than unity. This limit shows however that the importance of $\Delta E_{\mathrm{VdW}}$ increases as the long range Coulomb coupling raises towards the intermediate values. For such, physically realistic values of the Coulomb interaction, the two energies start to compete, in accordance with the simultaneous existence in nature of the TTF-TCNQ and HMTTFTCNQ lattices. This leads us to believe that the Coulomb energies of the metallic chains play a major role in the formation of the segregated stack lattices. On the other hand, other energies and in particular $E_{\mathrm{EC}}$ certainly control such details as e.g. the herring-bone stacking of the molecules.

Let us mention finally that the problem of the intermediate couplings can be treated somewhat more accurately than in the present treatment by using the bosonization method [11]. Indeed, with the backward interchain scattering neglected it is possible to extend the notion of the free plasmon beyond the range $|\mathbf{q}|<k_{\mathrm{TF}}$. Expressing the cohesive energy in terms of the zero point motion of such bosons extends the range of equation (3) to the non-perturbative region. This and other improvements of the present results are currently under investigation.

\section{References}

[1] See e.g. the review paper of André, J. J., Bieber, A. and Gautier, F., Ann. Phys. 1 (1976) 145.

[2] Denoyer, F., Comès, R., Garito, A. F. and Heeger, A. J., Phys. Rev. Lett. 35 (1975) 445 ; Kagoshima, S., Anzai, H., Kajimura, K. and Ishiguro, T., J. Phys. Soc. Japan 39 (1975) 1143.

[3] Megtert, S., Pouget, J. P., Comès, R., Garito, A. F., Bechgaard, K., Fabre, J. M. and Giral, L., J. Physique Lett. 39 (1978) L-118.

[4] Blessing, R. H. and Coppens, P., Solid State Commun. 15 (1974) 215.

[5] Metzger, R. M. and Bloch, A. N., J. Chem. Phys. 63 (1975) 5098.

[6] Epstein, A. J., Lipari, N. O., Sandman, D. J. and Nielsen, P., Phys. Rev. B 13 (1976) 1569.

[7] Klimenko, V. E., Krivnov, V. Ya., Ovchinnikov, A. A., Ukrainskit, I. I., and Shvets, A. F., $Z h$. Eksp. Theor. Fiz. 69 (1975) 240 (JETP 42 (1976) 123).

[8] Torrance, J. B. and Silverman, B. D., Phys. Rev. B 15 (1977) 788 ;

Silverman, B. D., ibid. 16 (1977) 5153.

[9] FrIedel, J., Electron Phonon Interactions and Phase Transitions, ed. T. Riste, NATO Advanced Study Institute, Series B Physics (Plenum, New York) 1977.

[10] Debray, D., Millet, R., Jérome, D., Barišić, S., Fabre, J. M. and Giral, L., J. Physique Lett. 38 (1977) L-227 ;

Bariší, S., J. Physique Colloq. 39 (1978) C2-263.

[11] EMERy, V. J., Highly Conducting One-Dimensional Solids, p. 247, ed. J. T. Devreese, R. P. Evrard and V. E. van Doren (Plenum, New York) 1979.

[12] Sоlyom, J., Adv. Phys. 28 (1979) 201.

[13] Barisite, S., J. Physique to be published (February 1983).

[14] Jacobsen, C. D., TANner, D. B. and BechgaARd, K., Phys. Rev. Lett. 46 (1981) 1142.

[15] Friedel, J., The Physics of Metals, Vol. I, ed. J. M. Ziman (Cambridge U.P., England) 1970.

[16] Barisić, S., Ann. Phys. 7 (1972) 23.

[17] Anderson, P. W. Concepts in Solids (W. A. Benjamin, New York, Amsterdam) 1964.

[18] Chang, D. B., Cooper, R. L., Drummond, J. E. and Young, A. C., Phys. Lett. 37A (1971) 311 ;

Davies, B., Ninham, B. W. and Richmond, P., J. Chem. Phys. 58 (1973) 744.

[19] Pines, D., Elementary Excitations in Solids (W. A. Benjamin Inc., New York, Amsterdam) 1964.

[20] Dzyaloshinskit, I. E. and Kats, E. I., Zh. Exp. Theor. Fiz. 55 (1968) 338 [Sov. Phys. JETP 28 (1969) - 178].

Williams, P. F. and Bloch, A. N., Phys. Rev. B 10 (1974) 1097.

[21] ŠAuB, K., BARIŠIĆ, S. and Friedel, J., Phys. Lett. 56A (1976) 302.

[22] Abramowitz, M. and Stegun, I. A., Handbook of Mathematical Functions (Dover, New York) 1972. 